\title{
A PET INVESTIGATION OF LEXICALITY AND PHONOTACTIC FREQUENCY IN ORAL LANGUAGE PROCESSING
}

\author{
Majerus $\mathrm{S}^{(1)}$,Collette $\mathrm{F}^{(1)}$ Van der Linden $M^{(1)(2)}$ Peigneux $P^{(1)}$, Laureys $S^{(1)}$, Delfiore $G^{(1)}$, \\ Degueldre $C^{(1)}$, Luxen $A^{(1)}$, Salmon $E^{(1)}$ \\ (1) University of Liège, Belgium \\ (2) University of Geneva, Switzerland
}

\begin{abstract}
Lexicality and phonotactic frequency effects are observed in many cognitive studies on language processing, but little is known about their underlying neural substrates, especially with regard to phonotactic frequency effects. Here, we conducted a positron emission tomography (PET) study in which 11 right-handed volunteers had either to repeat or to listen to lists ofwords, high phonotactic frequency nonwords, and low phonotactic frequency nonwords. The comparison of word versus nonword processing consistently confirmed previous findings of left temporal and prefrontal activations classically ascribed to lexicosemantic processing. Higher activation was found in the right posterior superior temporal gyrus when comparing high phonotactic frequency nonwords to words, but not when comparing low phonotactic frequency nonwords to words. We propose that this region is implicated in the formation of temporary phonological representations for high-probability phonological events, which may support processing of high phonotactic frequency nonwords.
\end{abstract}

\section{Introduction}

A number of studies in cognitive psychology and neuropsychology have shown an advantage in processing words compared to nonwords in speech perception and identification tasks (Pitt \& McQueen, 1998; Vitevitch \& Luce, 1998; Vitevitch \& Luce, 1999; Vitevitch, Luce, Charles-Luce, \& Kemmerer, 1997) as well as in verbal short-term memory tasks (Gathercole, Frankish, Pickering, \& Peaker, 1999; Gathercole, Hitch, Service, \& Martin, 1997; Hulme, Maughan, \& Brown, 1991). This advantage is considered to be due to the presence of lexical and semantic representations which underlie perception and short-term storage of words. Furthermore, nonwords, constructed by using phoneme associations that are frequent and highly probable (high phonotactic frequency) in a given natural language, are processed more quickly in shadowing and same-different judgment tasks as well as retained betterin short-term memory than nonwords composed of infrequent phoneme associations (Gathercole etal., 1999; Vitevitch \& Luce, 1998, 1999; Vitevitch et al., 1997). Similarly, 9-month-old infants prefer listening to monosyllables containing frequently rather than infrequently occurring phonetic patterns relative to their native language (Jusczyk, Friederici, Wessels, Svenkerud, \& Jusczyk, 1993; Jusczyk, Luce, \& Charles-Luce, 1994).

The nonword phonotactic frequency effect has been attributed to the existence of sublexical phonological knowledge, containing information about the statistical regularities and probabilities of phoneme associations in a given language, favouring the processing of nonword stimuli composed of more frequent phoneme sequences (Gathercole et al., 1999; Vitevitch \& Luce, 1998). A second interpretation for this effect considers that nonword familiarity stems from the similarity of the nonwords to known words, with high phonotactic frequency nonwords having more lexical neighbours (familiar words which share phoneme sequences with the nonwords) than low-frequency nonwords (Greenberg \& Jenkins, 1964; Luce \& Pisoni, 1998; Newman, Sawusch, \& Luce, 1996; Vitevitch, 1997). However, this supposed lexical influence on nonword processing might actually slow down the processing of high phonotactic frequency nonwords in certain conditions where lexical information is a determinant for the task to be processed. Indeed, Vitevitch and Luce (1999) showed that in lexical decision tasks, high phonotactic frequency nonwords are actually processed more slowly than low phonotactic frequency nonwords. This inversed phonotactic frequency effect for nonwords is explained by the activation of a greater number of lexical neighbours by high phonotactic frequency nonwords, which slows down lexical decision times. However, a normal phonotactic frequency effect for nonwords was observed when the same nonword stimuli were presented in a speeded identification 
task. According to Vitevitch and Luce (1999), these data suggest that the lexical and sublexical influence onnonword processing can be dissociated depending on the processing level which is required, with facilitatory effects for high phonotactic frequency nonwords when phonotactic, sublexical knowledge is used, and inhibitory effects when lexical knowledge is used.

Further, phonotactic frequency effects in the processing of words reflect influences of lexical neighbourhood: indeed, high phonotactic frequency words are processed more slowly in a speeded identification and in a lexical decision task, suggesting that more lexical competitors are activated when processing words containing high versus low phonotactic probability patterns, inhibiting processing of high phonotactic frequency words. Thus effects of phonotactic frequency operate in different ways, depending on the level of representation that dominates processing. Nonwords, when processed in shadowing and same-different judgment tasks, benefit from sublexical phonotactic knowledge. Words, and nonwords when processed in lexical decision tasks, are influenced by lexical competition rather than sublexical phonological knowledge.

Although lexicality and nonword phonotactic frequency seem to be reliable and well-investigated effects in cognitive studies, brain imaging studies appear less consistent in showing functional differences in brain activation for word versus nonword processing. Several studies observed activations in brain areas attributed to lexicosemantic processing when comparing words to nonwords. Left-hemispheric activations were found in the anterior superior temporal sulcus (BA 22/38), anterior middle (BA 21) and posterior inferior temporal gyri (BA 20/37), inferior parietal (BA 39, angular gyrus), and inferior frontal gyrus (BA 46/47) and dorsal prefrontal regions (BA 8/9/10); activations were also found in the right hemisphere in the posterior inferior temporal gyrus (BA 20/37) and the right angular gyrus (BA 39) (Binder et al., 1999; Binder, Frost, Hammeke, Rao, \& Cox, 1996; Démonet et al., 1992; Démonet, Price, Wise, \& Frackowiak, 1994; Howard et al., 1992; Perani et al., 1996; Price et al., 1996; Scott, Blank, Rosen, \& Wise, 2000). These regions are also partially consistent with data from brain-damaged patients.

Indeed, processing of lexicosemantic information, as evidenced by object naming, word repetition, and word comprehension tasks, is impaired in patients having left-sided or bilateral lesions including the middle temporal cortex, anterior and posterior infero-lateral temporal cortex, the temporal pole, frontoparietal areas, and the temporo-parieto-occipital junction (Caramazza \& Shelton, 1998; Damasio, Grabowski, Tranel, Hichwa, \& Damasio, 1996; De Renzi \& Lucchelli, 1994; Foundas, Daniels, \& Vasterling, 1998; Hart \& Gordon, 1990; Hillis \& Caramazza, 1991; Knott, Patterson, \& Hodges, 1997; McCarthy \& Warrington, 2001; Sacchett \& Humphreys, 1992; Silveri \& Gainotti, 1988; Warrington \& McCarthy, 1983, 1987; Warrington \& Shallice, 1984). These patients have a severe impairment in word processing, whereas nonword processing in speech perception and phonological tasks can be in the normal range (e.g., Hart \& Gordon, 1990; Knott et al., 1997). Lesions of prefrontal areas are, however, less frequently observed in patients with a lexico-semantic impairment.

Prefrontal activation observed in functional neuroimaging tasks comparing word to nonword processing might be related to retrieval, selection, and monitoring processes performed on semantic information, rather than to lexicosemantic representations per se (Fiez, 1997; Price, Indefrey, \& Van Turennout, 1999; Rapcsak \& Rubens, 1990; Swick, 1998).

Moreover, other functional neuroimaging studies did not always find significant differences in brain activation when comparing the processing of words to nonwords (Binder et al., 2000; Hirano et al., 1997; Wise etal., 1991). These divergent results can be at least partially explained by the requirements of the tasks used to elicit brain activation: The studies that reported a difference between word and nonword processing used rather complex and active tasks, in which subjects either had to perform phonological or semantic judgments (Binder et al., 1999; Démonet et al., 1992, 1994) or to listen to complex speech or "nonspeech" sentences and stories (Perani et al., 1996; Scott et al., 2000), or in which repetition of words was compared to passive listening to reversed words (Howard et al., 1992). Although these tasks may maximise the difference in brain activation between words and nonwords as theyrequire rather elaborate processing of the stimuli that are presented, they are also likely to engage processes other than linguistic ones, e.g., metalinguistic/executive processes in the case of phonological and semantic judgements and motor processes when comparing word repetition to nonword listening. Yet, methodological differences in the statistical analyses might also explain some of the diverging results between studies. For example, contrary to Price et al. (1996), Binder et al. (2000) did not report significant brain activation differences between passive listening to words and nonwords. A noticeable difference between the two studies is that Binder et al. reported their results after correction for multiple comparisons in the brain volume, whereas Price et al. used a region of interest (ROI) strategy which allows reporting results without further corrections. But actually, Binder et al. also found lefthemisphere activations at a lower, uncorrected statistical threshold in the inferior and posterior middle temporal gyri, in the angular gyrus and in the middle frontal gyrus, which minimises the differences 
between those two studies.

Another critical methodological factor is that the degree of dissimilarity between words and nonwords was not always controlled in the different studies. Indeed, when nonwords are very similar to words, it is not really surprising that they seem to activate similar brain regions. When presenting nonwords that consist of recorded words played backwards (Howard et al., 1992), it is not clear how distant this nonspeech is from intelligible speech, at least in psycholinguistic terms. Therefore, it appears important to control the degree of similarity between words and nonwords, by controlling the phonotactic frequency of the nonword phoneme sequences, in order to assess how likely the phoneme associations of the nonwords are in the native language of the participants. It might be that greater and more reliable differences in brain activation between words and nonwords would emerge ifnonwordswere verydistant from familiar words.

To our knowledge, no brain imaging study has directly investigated the neural bases of the phonotactic frequency effect in comparing nonwords with high and low phonotactic frequencies. Depending on the lexical or sublexical interpretation of the nonword phonotactic frequency effect, activation in brain regions implicated either in lexicosemantic processing or in phonological sublexical processing might be expected. We have seen that in lexical decision tasks, high phonotactic frequency nonwords are processed more slowly than their low-frequency counterparts, due to the activation of a larger number of lexical neighbours. In that case, the same temporal, parietal, and prefrontal regions subtending lexicosemantic representations for words should also be activated when comparing high to low phonotactic frequency nonwords. However, in the more typical case where high phonotactic frequency nonwords are processed faster than low phonotactic frequency nonwords, as is the case in simple nonword perception, repetition, and verbal short-term memory, sublexical phonological knowledge is supposed to be involved and thus brain regions implicated in sublexical phonological processing should be activated when comparing high to low phonotactic frequency nonwords. The most likely brain area subtending the processing of sublexical phonological knowledge seems to be the superior temporal area. Data from brain lesioned patients with "word sound deafness," a condition characterised by a selective impairment of speech sound discrimination, show damage to bilateral superiortemporal lobe areas. However, the impairment in sound discrimination is not always restricted to speech sounds, as these patients sometimes have additional difficulties in discriminating nonlinguistic acoustical information, suggesting a more general impairment in auditory discrimination, at least for some patients (Best \& Howard, 1994; Buchman, Garron, Trost-Cardamone, Wichter, \& Schwartz, 1986; Tanaka, Yamadori, \& Mori, 1987).

Functional neuroimaging studies also suggest that the superior temporal area is implicated in sublexical phonological processing. Scott et al. (2000) found an area in the posterior part of the left superior temporal sulcus that seems specifically to respond to phonetic information. This region responded specifically to speech, to spectrally rotated speech (which isunintelligible butpreserves phonetic features and some original intonation), to noise-vocoded speech (which is intelligible, but has very weak intonation), but not to spectrally rotated noise-vocoded speech (which is completely unintelligible, does not contain any phonetic information and does not sound like a human voice). The lowest common denominator of the three conditions to which the posterior superior temporal sulcus responded is precisely sublexical phonemic and phonetic information. Similarly, Mazoyer etal. (1993) also found left-lateralised posterior superior temporal gyrus activation when they presented a story spoken in an unfamiliar language (Tamil) to French subjects. Listening to an unfamiliar language like Tamil involves phonological processing of the phonemes that Tamil shares with French. However, since the word forms are very unfamiliar, theyare not likely to trigger lexicosemantic processing. Hence the left posterior superior temporal gyrus activation might be also related to sublexical phonological processing in the study by Mazoyer et al. Finally, Binder et al. (2000) found a bilateral superior temporal sulcus activation for passive listening to words, pseudowords, and reversed speech but not for frequency-modulated tones. What is common to the three speech conditions is that they contain phonological and phonetic information, but lexical-semantic content is greatly diminished for pseudowords and reversed speech. Frequency-modulated tones only share acoustic features with speech stimuli, but do not contain any phonological or phonetic features. In summary, brain imaging and functional neuroimaging studiesconcur to support the hypothesis that identification and processing of sublexical phonological information might be related to activation in the superior temporal gyrus. In the present PET study the neural bases of word and nonword processing were investigated using a paradigm in which the degree of similarity of the nonwords to familiar words was carefully controlled on the basis of phonotactic frequency. Two sets of nonwords were used: nonwords that were very close to existing words and whose phonotactic frequencies were matched to words,

and nonwordsvery distantfrom the words and from the first nonword set, with very low phonotactic frequencies. Furthermore, the three stimulus conditions were administered both in passive listening and 
repetition conditions.

A first aim was to investigate the neural basis of the lexicality effect, during repetition and passive listening, by contrasting words to high and low phonotactic frequency nonwords. We expected that words would activate areas implicated in lexicosemantic processing, i.e., middle and inferior temporal, parietal, and prefrontal regions, especially when compared to low phonotactic frequency nonwords. These differences in brain activation might be less important when comparing words to phonologically more familiar nonwords, i.e., the nonwords that are matched to the words on the basis of phonotactic frequency.

A second aim of the study was to investigate the neural basis of the phonotactic frequency effect. As the phonotactic frequency effect is quite complex and as PET studies only allow a limited number of conditions to be compared, we chose to restrict our study to the exploration of sublexical phonological influences on phonotactic frequency. Vitevitch and Luce $(1998,1999)$ have shown that sublexical phonological influences are implicated when using high and low phonotactic frequency nonwords and when comparing these nonwords in simple perception and repetition tasks. In this case high phonotactic frequency nonwords are processed faster and more accurately, as sublexical phonological knowledge is considered to support processing of nonwords containing high probability phoneme associations. We investigated the neural substrate of sublexical phonological influences on nonword phonotactic frequency by hypothesising that comparison of cerebral activation for high and low phonotactic frequency nonwords during repetition and passive listening conditions should evidence activation of left-sided or bilateral superior temporal brain regions implicated in sublexical phonological processing, but not inferior temporal, parietal and prefrontal brain regions implicated in lexico-semantic processing.

Thus, the design of this study allowed us to isolate the cerebral areas involved in lexicosemantic processing (words versus high phonotactic frequency nonwords and words versus low phonotactic frequency nonwords) and in sublexical phonological processing (high-frequency non-words versus low-frequency nonwords), independently of task-specific requirements (repetition or simple passive listening). Moreover, this study also enabled us to investigate brain regions activated in verbal repetition and passive listening (repetition versus listening) independently of the lexical and phonotactic status (words or nonwords) of the stimuli.

\section{Material and methods}

\section{Subjects}

Eleven French-speaking right-handed male volunteers (age 18-25 years) gave their written informed consent to take part in this study, which was approved by the Ethics Committee of the University of Liège. None had any remarkable past medical history nor did they use any medication.

\section{Cognitive tasks}

Three lists of bisyllabic stimuli were created: 80 low phonotactic frequency nonwords, 80 high phonotactic frequency nonwords, and 80 words. Each stimulus had the same syllabic structure CVCCVC. The low-frequency nonwords were constructed using CV and VC diphones, which are quite rare in French (e.g., in the nonword /fûgloS/, the diphones /fû/, /ûg/, /lo/, and /oS/ are not very frequent in French). On the other hand, the high-frequency nonwords contained $\mathrm{CV}$ and $\mathrm{VC}$ diphones which are quite frequent (e.g., in the nonword /pebmyn/, the diphones /pe/, /eb/, /my/, and /yn/ are very frequent in French). Furthermore, the diphones of the high-frequency nonwords had the same phonotactic frequency as the diphones of the words used in this study. The diphone frequencies were taken from a phonetic database of French by Tubach and Boë (Un corpus de transcription phonétique, 1990). This database was developed on the basis of a phonetic transcription of formal and informal conversations between French-speaking subjects. To measure phonotactic frequency, we used the simple diphone frequency counts listed in the database. There was a significant difference in summed diphone frequency counts for the $\mathrm{CV}$ and $\mathrm{VC}$ diphones in the first syllable ofthe high-frequency versuslowfrequency nonwords (2106 vs. 138), $F(1,158)=211.3, p<.0001$, and in the second syllable (2293 vs. $154), F(1,158)=320.9, p<.0001$; there was no difference in summed diphone frequency counts for the first syllable of high-frequency nonwords versus words (2106 vs. 2255), $F(1,158)=.549, p=.46$, and for the second syllable (2293 vs. 2345$), F(1,158)=0.09, p=.76$. Furthermore, the consonants and vowels used for the three lists were sampled from the same pool of phonemes.

The stimuli were recorded by a trained native French female speaker. All stimuli were spoken in isolation. The stimuli were low-pass filtered at $4.8 \mathrm{Khz}$ and digitised at a sampling rate of $11025 \mathrm{~Hz}$. 
All stimuli were edited into individual files and stored on computer disk. There were no significant differences in mean stimulus duration for the different stimuluslists: words vs. high phonotacticfrequency nonwords (1059vs. 1066ms), $F(1,158)<1, p=.68$, and high vs. low phonotactic frequency nonwords (1066 vs. $1079 \mathrm{~ms}), F(1,158)<1, p=.39$.

Stimulus presentation was controlled via E-Prime beta software (Psychology Software Tools, Inc., 2000) installed on a PC desktop computer. Stimuli were presented via headphones. Each subject was administered four blocks of 20 words, four blocks of 20 high phonotactic frequency nonwords, and four blocks of 20 low phonotactic frequency nonwords. For each condition, half of the blocks were presented for passive listening and half of the blocks were presented for repetition; blocks presented for repetition and passive listening within each word condition were alternated between subjects. In the passive listening trials, subjects were told that they would hear words or nonwords, and that they only had to listen to these stimuli. In the repetition trials, subjects were told that they had to repeat as accurately and as quickly as possible the words or nonwords that they were going to hear. Behavioural data were collected by recording the subjects' responses via a digital-to-analogue tape recorder connected to a microphone; latency times between the onset of the stimulus and the onset of the subjects' response were also recorded via a second microphone connected to E-Prime beta software. In each block, the stimuli were presented at a rate of one item every $4 \mathrm{~s}$. The order of presentation of the blocks and of the stimuli within each block was randomised. Before each block, the subjects were told by the experimenter that they would hear either words or nonwords and that they would have either to listen or to repeat. The subjects were explicitly informed about the lexical status of the stimuli they would hear in order to avoid lexical search processes when presenting nonwords while they might have been expecting to hear words.

\section{Positron emission tomography scanning}

PET data were acquired on a Siemens CTI 951 R 16/31 scanner in 3D mode. The subject's head was stabilised by a thermoplastic facemask secured to the head holder (Truscan imaging, MA), and a venous catheter was secured in a left antebrachial vein. First, a 20-min transmission scan was acquired for attenuation correction using three rotating sources of $68 \mathrm{Ge}$. Then, regional cerebral blood flow, taken as a marker of local neuronal activity (Jueptner \& Weiller, 1995), was estimated during 12 emission scans. Each scan consisted of two frames: a 30-s background frame and a 90 -s acquisition frame. The slow intravenous water $\left(\mathrm{H}_{2}{ }^{15} \mathrm{O}\right)$ infusion began $10 \mathrm{~s}$ before starting the second frame. Six $\mathrm{mCi}(222 \mathrm{MBq})$ in $5 \mathrm{cc}$ saline were injected over a period of $20 \mathrm{~s}$ for each scan. The infusion was totally automated in order not to disturb the subject during the scanning period. Data were reconstructed using a Hanning filter (cut off frequency: .5 cycle/pixel) and corrected for attenuation and background activity. Each experimental task (block) corresponded to one scan acquisition and began $10 \mathrm{~s}$ before starting the acquisition frame.

\section{Data analysis}

Data were analysed and transformed using the statistical parametric mapping software (SPM99; Wellcome Department of Cognitive Neurology, Institute of Neurology, London, UK) implemented in MATLAB (Mathworks Inc., Sherborn, MA). First, spatial transformations were performed to accommodate within-subject head-movement in scan replications and inter-subject differences in gyral and functional anatomy. For each subject, all scans were realigned together, then normalised to a standard PET template (Friston et al., 1995). Spatial registration and normalisation of images conform to the space defined by the ICBM, NIH P-20 project, and approximate that of the space described in the stereotaxic atlas of Talairach and Tournoux (1988). Finally, PET images were smoothed using a Gaussian kernel of $16 \mathrm{~mm}$ full width at half maximum.

\section{Random-effect analyses}

First, we looked for the cerebral areas specifically and consistently supporting the task effect (repetition [R] vs. passive listening [L]), independently of the stimulus condition (i.e., words [W], high phonotactic frequency nonwords [HN], or low phonotactic frequency nonwords [LN]). Next, the analysis aimed to evidence stimulus-related effects independently of the task condition (i.e., repetition or passive listening): word vs. high-frequency nonword processing [W vs. HN], word vs. low frequency nonword processing [W vs. LN], and high vs. low phonotactic frequency nonword processing [HN vs. LN]. These analyses were conducted using the random effect model (Holmes \& Friston, 1998) implemented in SPM99 in order to specify the cerebral areas activated in each of our 11 
subjects. The random effect model is a two-step procedure, based on the approach of mean summary statistics on repeated measures (Frison \& Pocock, 1992), applied to accommodate both interindividual and intraindividual variability of PET data. It requires that all members of the population show this effect such that its expectation is greater than under the null hypothesis.

At the first step, PET data were analysed separately at a within-subject level using standard subtraction analyses. Because a separable model is required to allow subsequent modelling of the between-subject variance, the adjustment for one subject was made independent from other subjects by using ANCOVA adjustment of the global activity. Three contrasts estimated the task effect in each subject within each stimulus condition: repetition vs. listening of words (W [R vs. L]), high phonotactic frequency nonwords (HN [R vs. L]), low phonotactic frequency nonwords (LN [R vs. L]). Three contrasts estimated the stimulus effect during repetition: word vs. high-frequency nonword (R [W vs. HN]), word vs. low-frequency nonword (R [W vs. LN]), and high-frequency nonword vs. low-frequency nonword ( $\mathrm{R}[\mathrm{HN}$ vs. LN]). The three remaining contrasts estimated the stimulus effect during listening: word vs. high-frequency nonword (L [W vs. HN]), word vs. low-frequency nonword (L [W vs. LN]), and high-frequency nonword vs. low-frequency nonword (L [HN vs. LN]). The resulting estimates (contrast images) fitted the within-subject component of the variance and could then be used for a subsequent second-level analysis, in which the between-subject variance is taken into account. At the second level, conjunctions were performed between identical contrasts across stimuli (W\&HN \&LN [Rvs. L]) or tasks (R \&L [Wvs. HN]; R \& L [W vs. LN]; R \& L [HN vs. LN]) to highlight the brain areas whose activity specifically subtended the processing of each task condition independently of stimulus type, or of each stimulus type independently of the task condition. The resulting set of voxel values for each contrast constituted a map of the $t$ statistic, $\operatorname{SPM}(\mathrm{T})$, thresholded at $p<.001$ (T> 1.31). If not otherwise specified, statistical inferences were obtained at the voxel level (in terms of peak height at $p<.05$ ), corrected for multiple comparisons. The significance was set at the level of $p$ (uncorrected) $<.001(T>1.31)$ when we had an a priori hypothesis concerning the brain areas of interest, based on brain activations previously described in the literature. These were the bilateral precentral gyrus, left inferior frontal gyrus, middle and superior temporal areas, insula, putamen, thalamus, anterior cingulate, and cerebellum for the comparison between repetition and passive listening (Howard et al., 1992; Karbe, Herholz, Weber-Luxenburger, Ghaemi, \& Heiss, 1998; Petersen, Fox, Posner, Mintun, \& Raichle, 1988, 1989; Price et al., 1996, 1999), and the left anterior superior temporal sulcus (BA 22/38), anterior middle (BA 21)and posterior inferior temporal gyri (BA 20/37), inferior parietal (BA 39, angular gyrus) and inferior frontal gyrus (BA 46/47), and dorsal prefrontal regions (BA 8/9/10), as well as the right posterior inferior temporal gyrus (BA 20/37) and angular gyrus (BA 39) for word compared to nonword processing (Binder et al., 1996, 1999, 2000; Démonet et al., 1992, 1994; Howard et al., 1992; Perani et al., 1996; Petersen et al., 1988, 1989; Price, Wise, \& Frackowiak, 1994; Price etal., 1994, 1996; Scott et al., 2000).

\section{Results}

\section{Behavioural data}

Mean latency times and response accuracy were analysed for the repetition of words, high phonotactic frequency nonwords, and low phonotactic frequency nonwords. Two separate repeated measures ANOVAs were performed for mean latency times and for response accuracy.

Asexpected, mean latencytime was significantly lower for words vs. high phonotactic frequency nonwords (1297 vs. $1465 \mathrm{~ms}), F(1,11)=55.96, p<.0001$, and for high vs. low phonotactic frequency nonwords (1465 vs. $1531 \mathrm{~ms}) ; F(1,11)=6.33, p<.05$. Accuracy was globally very high (mean accuracy: .95). Accuracy was also higher for the repetition of words compared to high phonotactic frequency nonwords (.99 vs. .96), $F(1,11)=24.27, p<.01$, and for high phonotactic frequency nonwords compared to low frequency nonwords (.96 vs. .91), $F(1,11)=19.46, p<.01$.

\section{Imaging data}

Task effect : Repetition vs. passive listening

The random effect conjunction analysis $[\mathrm{W}(\mathrm{R}-\mathrm{L})] \&[\mathrm{HN}(\mathrm{R}-\mathrm{L})] \&[\mathrm{LN}(\mathrm{R}-\mathrm{L})]$ showed the brain areas in which rCBF increased during repetition as compared to listening, independently of the stimulus type (words, high or low phonotactic frequency nonwords). The analysisshowed significant increases bilaterally in the precentral gyrus (BA 4/6), the anterior part of the superior temporal lobe (BA 22/42), the thalamus, and the cerebellum. Additional activation foci were found in the right insula 
and the right putamen. Activation at uncor-rected $p$ levels was observed in a priori regions of interest : the left inferior frontal gyrus (BA 47) close to Broca's area, and the left anterior cingulate (BA 32).

(See Table 1, and Plate 1 situated between pp. 352 and 353.)

The reverse comparison $[\mathrm{W}(\mathrm{L}-\mathrm{R})] \&[\mathrm{HN}(\mathrm{L}-\mathrm{R})] \&[\mathrm{LN}(\mathrm{L}-\mathrm{R})]$ showed significant $(p<.05$, corrected) activation in the left middle and superior frontal gyri (BA 9/10/11), in the left medial frontal gyrus (BA 10), the right superior frontal gyrus (BA 8), the left posterior middle temporal gyrus (BA 21), the left inferior parietal gyrus including the angular gyrus (BA 39), the bilateral middle occipital gyrus (BA 19), as well as the right superior occipital gyrus (BA 19) and right praecuneus (BA 7). (See Table 1, and Plate 1 situated between pp. 352 and 353.)

Stimulus effect: Words vs. high-frequency nonwords

The random effect conjunction analysis $[\mathrm{R}(\mathrm{W}-\mathrm{HN})] \&[\mathrm{~L}(\mathrm{~W}-\mathrm{HN})]$ showed the brain areas in which rCBF increased during word as compared to high phonotactic frequency nonword processing, independently of the task to be performed. No voxel survived correction for multiple comparisons. At $p$ $<.001$ (uncorrected), increased activations were found in a priori regions of interest: the anterior part ofthe left superior temporal gyrus (BA 38), as well as the left fusiform gyrus (BA 37/20) in the posterior inferior temporal region (see Table 2).

The reverse comparison $[\mathrm{R}(\mathrm{HN}-\mathrm{W})] \&[\mathrm{~L}(\mathrm{HN}-\mathrm{W})]$ disclosed significant $(p<.05$, corrected) activation in the right posterior superior temporal gyrus (BA 22). (See Table 3, and Plate 2 situated between pp. 352 and 353).

Stimulus effect: Words vs. low-frequency nonwords

The random effect conjunction analysis $[\mathrm{R}(\mathrm{W}-\mathrm{LN})] \&[\mathrm{~L}(\mathrm{~W}-\mathrm{LN})]$ showed the brain areas in which $\mathrm{rCBF}$ increased during word as compared to low phonotactic frequency nonword processing, independently of the task to be performed.

No voxel survived correction for multiple comparisons. At $p<.001$ (uncorrected), increased activations were found in a priori regions: left fusiform gyrus in the posterior inferior temporal region (BA 20/37), as well as inferior and superior prefrontal regions (BA 47, BA 10) (see Table 2).

The reverse comparison $[\mathrm{R}(\mathrm{LN}-\mathrm{W})] \&[\mathrm{~L}(\mathrm{LN}-\mathrm{W})]$ failed to disclose significant activation foci. Stimulus effect: High- vs. low-frequency nonwords

The random effect conjunction analysis $[\mathrm{R}(\mathrm{HN}-\mathrm{LN})] \&[\mathrm{~L}(\mathrm{HN}-\mathrm{LN})]$ showed the brain areas in which rCBFincreased duringhigh phonotactic frequency nonword as compared to low phonotactic frequency nonword processing, independently of the task to be performed. No voxel survived the activation threshold (corrected and uncorrected). The same lack of significant effect was observed for the reverse comparison $[\mathrm{R}(\mathrm{LN}-\mathrm{HN})] \&[\mathrm{~L}(\mathrm{LN}-\mathrm{HN})]$. 
Table 1. Conjunction analysis comparing repetition to passive listening for words, high phonotactic frequency nonwords and low phonotactic frequency nonwords (random effect model)

\section{St ereotaxic coordinates}

Brain area

T-value Brain area

Stereotaxic coordinates $\begin{array}{llll}x & y & z & T\end{array}$ valu
Repetition-Passive

listening

Precentral gyrus L

(BA 4, 6)
Passive listening -

Repetition

Superior frontal gyrus L $\quad-16 \quad 44 \quad 29 \quad 4.10$

(BA 9)

Superior frontal gyrus L $-22 \quad 59 \quad 17 \quad$\begin{tabular}{llll}
3.73 \\
\hline
\end{tabular}

(BA 10)

L

(BA 47)

Precentral gyrus R $\quad 50 \quad-14 \quad 32 \quad 6.22$

(BA 4, 6)

Insula $\mathrm{R}$

$\begin{array}{llll}28 & -13 & 14 & 4.18 *\end{array}$

Superior temporal $\quad-65 \quad-5 \quad 8 \quad 5.24 *$

gyrus

L (BA 22)

Superior temporal $\quad-69-17 \quad 6 \quad 4.16^{*}$ gyrus

L (BA22/42)

Superior temporal

gyrus

R (BA 22)

Cingulate gyrus L $\quad-10 \quad 23 \quad 32 \quad 2.30 * *$

(BA 32)

Thalamus $\mathrm{R}$

$8 \quad-21 \quad-2 \quad 4.92 *$

Cerebellum L

$\begin{array}{llll}-4 & -61 & -17 & 5.03 *\end{array}$

$\begin{array}{llll}-12 & -57 & -14 & 4.57 *\end{array}$

Middle frontal gyrus L

$\begin{array}{llll}-32 & 54 & -3 & 3.88 \\ *\end{array}$

(BA 10/11)

$\begin{array}{lllll}\text { Medial frontal gyrus R } & 28 & 31 & 43 & 2.67\end{array}$

(BA 8)

$\begin{array}{lllll}\text { Middle temporal gyrus L } & -51 & -20 & -17 & 3.21\end{array}$

(BA 21)

$\begin{array}{lllll}\text { Inferior parietal gyrus L } & -38 & -70 & 27 & 5.57\end{array}$

(BA 39)

Middle occipital gyrus L $\quad-44 \quad-81 \quad 10 \quad 3.81$

(BA 19)

$\begin{array}{lllll}\text { Superior occipital gyrus } & 40 & -76 & 28 & 3.48\end{array}$

$\mathrm{R}$

(BA 19)

Middle occipital gyrus R $\quad 48 \quad-79 \quad 11 \quad 3.81$

(BA 19)

Praecuneus R (BA 7) $\quad 2 \quad-54 \quad 40 \quad 3.42$

Cerebellum R $\quad 28 \quad-63 \quad-15 \quad 5.41 *$

$12-63-15 \quad 4.23 *$

Coordinates and T-values for voxels in which there was significant activation when repetition was compared to passive listening of words, high phonotactic frequency nonwords, and low phonotactic frequency nonwords. $L=$ left hemisphere; $R$ =right hemisphere. $x, y, z$ (in $\mathrm{mm}$ ) refer to coordinates in the Talairach and Tournoux stereotaxic space (1988). ${ }^{*} p<.05$, corrected for multiple comparisons; $* * p<.001$, uncorrected.

\section{Discussion}

In this functional neuroimaging study, we investigated brain activation associated with lexicality and nonword phonotactic frequency effects in passive listening and repetition conditions.

We will first discuss brain activation associated with the task demands, repetition versus passive listening, and then discuss activation associated with lexicality and nonword phonotactic frequency effects.

Brain activation associated with repetition and passive listening tasks

When comparing repetition of words and nonwords to passive listening, we identified bilateral activity 
in the perisylvian area including the precentral gyrus and the anterior part of the superior temporal gyrus; the thalamus and the cerebellum were also activated bilaterally as well as the right insula and the right putamen. Weaker activation was found in the left inferior frontal gyrus and the anterior cingulate cortex. These regions have classically been identified as reflecting the cerebral network implicated in phonological output processes and motor control ofverbal response production (Howard et al., 1992; Karbe et al., 1998; Petersen et al., 1988, 1989; Price et al., 1996). The precentral, medial frontal, cerebellar, and sub-cortical brain regions are involved in the planning and control of motor output processes of the organs implicated in speech production (Howard et al., 1992; Price et al., 1999).

Table 2. Conjunction analysis comparing word to low and high phonotactic frequency nonword processing for repetition and passive listening (random effect model)

\section{Brain area}

Words-High phonotactic frequency nonwords

Fusiform gyrus L (BA 37)

Fusiform gyrus L (BA 20)

Superior temporal gyrus L (BA 38)

Words-Low phonotactic frequency nonwor $d s$

Inferior temporal gyrus L (BA 20)

Fusiform gyrus L (BA 37)

Superior frontal gyrus L (BA 10)

Inferior frontal gyrus L (BA 47)
Stereotaxic coordinates

$-50 \quad-56$

$-30 \quad-32$

14

$-17$

$-21$

17

$-10$

15

$-12$

$-49 \quad 25$

Coordinates and T-values for voxels in which there was significant activation when words were compared to low phonotactic frequency nonwords for repetition and passive listening. $\mathrm{L}=$ left hemisphere ; $\mathrm{R}=$ right hemisphere. $\mathrm{x}, \mathrm{y}, \mathrm{z}$ (in $\mathrm{mm}$ ) refer to coordinates in the Talairach and Tournoux stereotaxic space (1988). **All $\mathrm{p}<.001$, uncorrected.

Table 3. Conjunction analysis comparing high and low phonotactic frequency nonword to word processing for repetition and passive listening (random effect model)

\section{Brain area} Stereotaxic coordinates

High phonotactic frequency nonwords-words

Superior temporal gyrus R (BA 22)

$x$

$y$

$z$

T-value

Low phonotactic frequency nonwords - words

49

$-32$

18

$3.80 *$

High-low phonotactic frequency nonwords

No significant activation

No significant activation

Coordinates and T-values for voxels in which there was significant activation when high and low phonotactic frequency nonwords were compared to words for repetition and passive listening. $R$ =right hemisphere. $x, y, z$ (in mm) refer to coordinates in the Talairach and Tournoux stereotaxic space (1988). ${ }^{*} p<.05$, corrected for multiple comparisons.

The superior temporal gyrus has been implicated in constructing sound-based representations ofspeech production (Binder et al., 2000; Hickok, 2000; Hickok et al., 2000; Hickok \& Poeppel, 2000; Price et al., 1999). More interestingly, our results suggest that the bilateral anterior superior temporal gyrus, identified in the present and previous studies as supporting phonological output processes, must contain sublexical rather than lexical phonological codes for speech output, as this region was activated for the repetition of both words and nonwords. More generally, our study shows that the cerebral network of speech production identified in this study is independent of the lexical status of verbal stimuli to be repeated, as these regions were activated equally for words and high or low phonotactic frequency nonwords. Our random effect analysis furthermore allows generalisation of these results to all members of the normal young population.

Relatively weak activation was observed in the inferior prefrontal cortex close to Broca's area. Although this region is classically considered to be very important for speech output processes, its precise role in language processing is still not very clear. Our results are also concordant with other studies which did not find consistent activation of Broca's area when comparing word repetition to a control condition (Herholz et al., 1994; Howard et al., 1992; Karbe et al., 1998). Recent data suggest that Broca's areamaybe implicated inspeech perception and production only when rehearsal and/or segmentation of the phonological information is needed, for example in phonological judgment tasks where the phonological form of the word has to be decomposed, segmented, and rehearsed (Burton, Small, \& Blumstein, 2000; Cabeza \& Nyberg, 2000; Démonet et al., 1992, 1994; Price et al., 1996; Zatorre, Evans, Meyer, \& Gjedde, 1992; Zatorre, Meyer, Gjedde, \& Evans, 1996). Some studies also 
suggest that Broca's area may be implicated in non-linguistic processing. This area has been shown tobe involved in nonlinguistic discrimination tasks as well as in visuomotor activities such as imagery of motion (Binkofski et al., 2000; Müller, Kleinhans, \& Courchesne, 2001).

The reverse contrast of passive listening minus repetition showed that during passive listening, activation was observed in mainly dorsal prefrontal regions as well as in temporo-parieto-occipital regions. These regions might reflect the greater possibility of reasoning, mental imagery and retrieval of lexicosemantic information while the participants are waiting for presentation of the next stimulus in the passive listening condition, whereas in the repetition condition, the major part of the inter-stimulus interval is occupied by the production of the stimuli. Indeed, the prefrontal regions and the temporoparieto-occipital regions have been associated with retrieval of semantic and associated visual information during the resting state (e.g., Binder et al., 1999). This furthermore shows that passive listening, although seemingly a quite simple task, is not necessarily a very "pure" activation task in the sense that it may generate experimentally uncontrolled cognitive processing.

\section{Brain activation associated with the lexicality effect}

When comparing processing of words and nonwords, no greater activation was observed for words vs. high and low phonotactic frequency nonwords at corrected threshold levels. However, at uncorrected thresholds, we identified regions in the left postero-inferior temporal lobe as well as in the left anterior superior temporal lobe that were specifically associated with word vs. high phonotactic frequency nonword processing. The same was true when comparing words to low-frequency nonwords, where activation was observed in the left postero-inferior temporal region, in addition to inferior and superior frontal activation. These results are consistent with Binder et al.'s (2000) study, which also found differences between words and nonwords only at uncorrected $p$ thresholds. The activation foci found in this study are very similar to the activation points that have been associated with lexicosemantic processing in other studies (Binder, 1999, 2000; Démonet et al., 1994; Howard et al., 1992; Perani et al., 1996; Petersen et al., 1988, 1989; Price et al., 1994, 1996; Scott et al., 2000). Furthermore, the fusiform area (BA 20/37) was consistently activated when comparing words to high and low phonotactic frequency nonwords. However, contrary to our hypotheses, the fact that we had controlled the degree of similarity of the nonwords to the words by creating two contrasted sets of nonwords on the basis of phonotactic frequency did not greatly influence brain activation: Differences in activation between words and nonwords were not more significant for low than for high phonotactic frequency nonwords; only some prefrontal activation was observed for words when compared to low but not to high phonotactic frequency nonwords. These data suggest that areas implicated in lexicosemantic processing were similarly activated when comparing words to high or to low phono-tactic frequency nonwords, suggesting that areas implicated in lexicosemantic processing are less active to a similar extent for both high and low phonotactic frequency nonwords. This finding is of theoretical importance regarding the lexical or sublexical interpretation of the nonword phono-tactic frequency effect and will be commented on in the last section of this discussion.

Overall, activation of regions subtending lexico-semantic processing was not very strong, as it was only significant at uncorrected statistical thresholds. A possible explanation is that regions implicated in lexicosemantics are permanently active to some extent during the wakeful resting state and also during listening and repetition of nonwords, independently of the stimuli that are presented. Indeed, Binder et al. (1999) showed that left-hemisphere brain areas implicated in semantic processing (angular gyrus, prefrontal cortex, ventral temporal cortex) were active during a wakeful resting state compared to a nonlinguistic tone discrimination task. As mentioned earlier, this baseline brainactivity might reflect our engagement in mental activities such as reasoning, problem solving, and memory retrieval of verbal information during quiet wakefulness. These processes might also be partially active during listening and repetition of nonwords, where subjects can reason and retrieve verbal information about the task and the stimuli to be performed. This might raise the baseline level of activation of regions implicated in lexicosemantic processing, and thus differences in lexicosemantic activation between word and nonword processing will be more subtle and more difficult to observe.

\section{Brain activation associated with the nonword phonotactic frequency effect}

Regarding the phonotactic frequency effect, we observed, when comparing nonwords to words, that high phonotactic frequency nonword processing activated aposterior superior temporal region in the right hemisphere, close to the planum temporale. This activation was not consistently observed when comparing low-frequency nonwords to words. We might then expect that the right posterior temporal gyrus (STG) is also activated when directly comparing high to low phonotactic frequency nonwords. 
However, the direct comparison between high and low phonotactic frequency nonwords failed to disclose any significant activation foci. This pattern of results can best be explained by the nature of our statistical analysis. We used a random effect analysis, which is based on activation foci that, in each subject, are systematically present in one condition and absent in the other. Therefore it is possible that the direct comparison between high and low phonotactic frequency nonwords did not show significant activation in the right superior temporal lobe, because some subjects also activated this brain area for low phonotactic frequency nonwords. This possibility was verified by an inspection of the individual activation maps contrasting low phonotactic frequency nonwords to words, on a subjectby-subject basis; this showed that for the low phonotactic frequency nonwords, 6 out of 11 participants also presented weak activations of the right posterior STG. At variance, all 11 subjects showed highly consistent and strong activation in the same brain area for high phonotactic frequency nonword processing, when compared to words.

How can this right posterior STG activation for high phonotactic frequency nonwords be explained? Beginning with our behavioural data, sublexical rather than lexical knowledge seems to have influenced nonword performance as high phonotactic frequency nonwords were processed faster and more accurately than low phonotactic frequency nonwords. Vitevitch and Luce (1999) predicted this advantage for high phonotactic frequency nonwords when sublexical knowledge is involved but an advantage for low phonotactic frequency nonwords when lexical knowledge is involved. This is also confirmed by our neuro-imaging data comparing lexicality effects for high and low phonotactic frequency nonwords, which have shown that brain areas implicated in lexicosemantic processing are less activated to a similar extent for both high and low phonotactic frequency nonwords compared to words, suggesting that there was no difference in lexicosemantic processing between the nonword conditions. Regarding imaging data and sublexical phonological processing, we had expected bilateral or left-sided activation of the STG but not only right-sided activation ofthe posterior STGfor processing high phonotactic frequency nonwords. This exclusively right-sided activation of the STG makes an interpretation of this area as reflecting activation of sublexical phonological knowledge unfortunately less likely. What are alternative interpretations for activation of the right posterior STG when processing high phonotactic frequency nonwords compared to words?

Price et al. (1994) observed greater activation in right and left temporal areas when comparing pseudowords to words in a nonlinguistic visual feature detection task. They attributed this activation to the fact that pseudowords activate phonological and semantic processing areas more than words because a more prolonged search for the unavailable representation occurs. In particular, a longer search in the phonological processing areas would be observed for the pseudowords which have unfamiliar phoneme associations. However, this alternative interpretation does not seem to match our data. First, we did not observe more activation for non-words in the entire cerebral network, which normally sustains semantic and phonological processing; the activation we observed was spatially limited to the right posterior superior temporal lobe. Second, if this activation reflected a more prolonged search for phonological representations due to nonwords having unfamiliar phonological associations, then this activation should have been greatest for the most unfamiliar phonological associations, namely our low phonotactic frequency nonwords. In fact, the opposite was observed. Further, if the activation observed for high phonotactic frequency nonwords in the right STG is related to phonological search processes, then behavioural data should also evidence these search processes, by a slower processing of high phonotactic frequency nonwords. However, our behavioural data clearly show that processing of high phonotactic frequency nonwords is facilitated rather than inhibited, compared to low phonotactic frequency nonwords, thus making an interpretation of the right posterior STG in terms of phonological search processes rather unlikely.

Alternatively, the activation in the right STG could reflect processes related to differences in pitch or task difficulty between word and nonword processing. These possibilities must, however, also be rejected as our three stimuli lists were carefully controlled with regard to phoneme composition, number of syllables, and syllabic structure, making a difference in pitch and intonation highly implausible. Furthermore, behavioural data showed that low phonotactic frequency nonwords were more difficult to process, as evidenced by greater reaction times and lower response accuracy in the repetition condition. Should right STG activation reflect this difference in difficulty, then a greater activation should be observed for low phonotactic frequency nonwords but not for high phonotactic frequency nonwords compared to words, which once again is clearly rejected by our data.

Another alternative interpretation to be considered are lexical competition processes for words. Indeed, presentation of a word stimulus does not only activate the corresponding lexical representation, but lexical neighbours are also activated, especially if the words are composed of high phonotactic frequency patterns, as Vitevitch and Luce $(1998,1999)$ have shown. These lexical competitors must be inhibited and the correct lexical representation has to be selected, a process which might be related to 
the right STG. This possibility is supported by studies of right-hemisphere-damaged patients, showing that these patients have difficulty in suppressing inappropriate lexico-semantic competitors when interpreting ambiguous sentences (Tompkins, Baumgaertner, Lehman, \& Fassbinder, 2000; Tompkins, Lehman, \& Fassbinder, 1998; Zaidel, Zaidel, Oxbury, \& Oxbury, 1995). However, if the right posterior STG reflects lexical selection and inhibition processes when processing words, than it should be more activated for word than for nonword stimuli. Clearly, this was not the case in our study.

Alternatively, this region might reflect lexical inhibition mechanisms for high phonotactic frequency nonwords that might also tend to activate lexical representations more than low phonotactic frequency nonwords. However, this is not very likely, as lexical inhibition should slow down responses to high phonotactic frequency nonwords compared to low phonotactic frequency nonwords, which clearly was not the case, as we have already discussed. Furthermore, the advantage in processing high over low phonotactic frequency nonwords suggests that sublexical phonological and not lexical processes influenced performance, according to Vitevitch and Luce (1998, 1999). Thus inhibition of lexical competitors either for words or high phonotactic frequency nonwords does not seem to be a likely explanation of the activation of the right posterior STG when comparing high phonotactic frequency nonwords to words.

The alternative interpretations for the right posterior STGactivation we have considered until now have all been rejected, mainly because they are not compatible with our behavioural results, which show facilitation for processing of high phonotactic frequency nonwords, while the interpretations we have considered predictan inhibitionfor processing of high phonotactic frequency nonwords or no difference between the two nonword conditions. How can the posterior STG facilitate processing of high phonotactic frequency nonwords, if this region cannot be considered as representing sublexical phonological knowledge that is supposed to favour processing of these nonwords?

A possible explanation is that the right posterior STG is able to build up temporary representations for phonological forms that are unknown, but which nevertheless are highly probable; this is the case for high but much less so for low phonotactic frequency nonwords. These temporary representations for high probability phonological forms then represent an interface representation supporting perception and repetition for high phonotactic frequency phonological forms, and thus favouring processing of high over low phonotactic frequency nonwords. Indeed, several studies have proposed that the bilateral posterior STG can construct temporary representations of high probability phonological events (Grasby et al., 1993; Hughes et al., 2001; Winkler, Karmos, \& Näätänen, 1996; Wise et al., 2001). The most convincing data come from the study by Hughes et al. These authors repeatedly presented the same CV syllable at regular ISI to epileptic patients, thus creating a high probability phonological event; sometimes, the CV syllable did not occur although it was expected. Intracranial ERP recordings showed that the posterior part of the bilateral superior temporal gyrus (STG)specifi-cally responded to the repetition of CV syllables, and, most importantly, was activated at the moment when the CV was expected to occur, even when it was not presented. This response to expected, but not necessarily presented, phonological information suggests that the posterior part of the STG had stored a temporary representation of the phonological event which predicted the occurrence of phonological information. This temporary representation would then serve to support perception, repetition, and other forms of processing for high-probability phonological information like the high phonotactic frequency nonwords in our study. Although words also represent high probability phonological information, there is less need to construct a temporary phonological information as word forms very quickly activate their corresponding long-term phonological representations, which support further processing.

But why was it specifically the right and not bilateral posterior STG that responded to high phonotactic frequency nonwords? A possible explanation is that right-hemisphere language areas, including the right STG, conserve a greater plasticity for language processing, and then respond more easily to new phonological information than their left-hemisphere counterparts. For example, Dehaene et al. (1997) showed that when adults were listening to a story in a second language acquired after the age of 7 years, compared to listening to the same story in their first language, they activated both left- and righthemisphere regions, and in some subjects exclusive activation of right temporal regions, including the middle and superior temporal gyri, was observed. These data suggest that processing of phonological information acquired after the critical period for phonological learning has a tendency to preferentially activate right-hemisphere language areas. This is also supported by studies of recovery from aphasia following lesions in left temporoparietal areas. For example, Musso et al. (1999) showed in a functional neuroimaging study that performance increase in a language comprehension task (shortened form of the Token Test) correlated with activation increase in the right posterior STG of lefthemisphere-damaged patients with Wernicke's aphasia, after a very short language therapy which targeted phonological and semantic processing. Although several cognitive mechanisms might have led 
to improved performance in the language comprehension task due to the short language therapy, it is clear that performance on a language comprehension task, like the Token Test, requires temporary storage of phonological information; the correlation between performance on the language comprehension task and the right posterior STG might thus be due to the involvement of temporary storage processes of the phonological information necessary to perform the comprehension task. Indeed, the studies presented here have shown that this region can construct temporary phonological representations supporting further processing of these representations, as is necessary in tasks like the Token Test. The study by Musso et al. (1999) furthermore suggests that the right posterior STG may exclusively support the formation of temporary phonological representations in certain conditions, at least following left-hemisphere brain lesions. Together with our results, these data support the idea that the right posterior STG is implicated in the construction of temporary phonological representations for high probability phonological events.

In conclusion, we have compared processing of words to high and low phonotactic frequency nonwords in passive listening and repetition conditions. We have shown that regions implicated in lexical and semantic processing were activated when comparing words to nonwords. Furthermore, high phonotactic frequency nonwords activated the right posterior STG more than words. We suggest that this region is implicated in the formation of temporary phonological representations for highprobability phonological events, which may support processing of high phonotactic frequency nonwords.

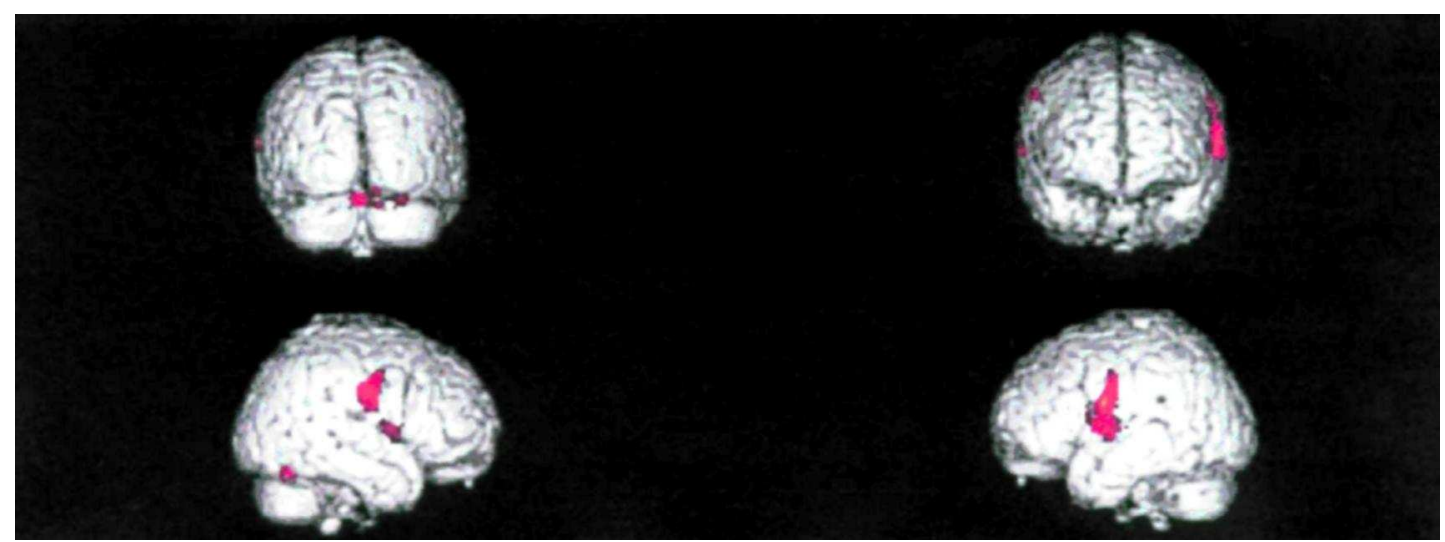

Plate 1. Cortical brain activation (corrected for multiple comparisons) observed when repetition was compared to passive listening, independently of stimulus condition (words or nonwords). Coordinates of all significant regions are given in Table 1. Brain areas are rendered on a standard 3D brain MRI.

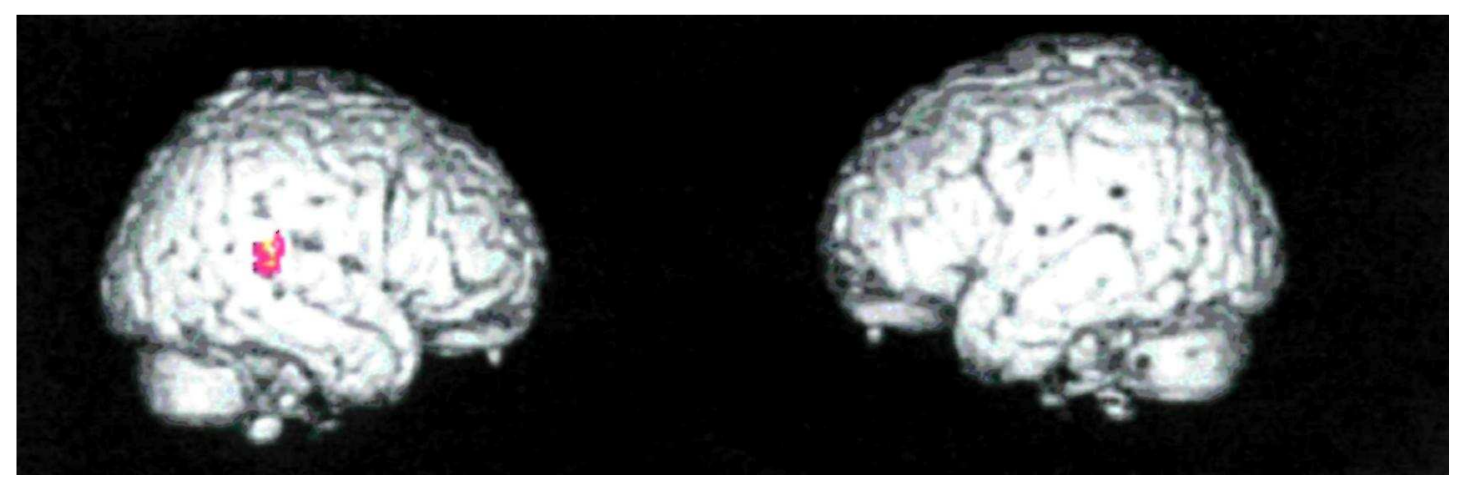

Plate 2. Brain activation (corrected for multiple comparisons) observed when high phonotactic frequency processing was compared to word processing, independently of task condition. Coordinates of all significant regions are given in Table 3. Brain areas are rendered on a standard 3D brain MRI. 
Published in: Cognitive neuropsychology (2002), vol. 19, iss. 4, pp. 343-360

Status: Postprint (Author's version)

\section{References}

Bailey, T.M., \& Hahn, U. (2001). Determinants of wordlikeness: Phonotactics or lexical neighbourhoods? Journal of Memory and Language,44, 568-591.

Best, W., \& Howard, D. (1994). Word sound deafness resolved? Aphasiology, 8, 223-256.

Binder, J.R., Frost, J.A., Hammeke, T.A., Bellgowan, P.S.F., Rao, S.M., \& Cox, R.W. (1999). Conceptual processing during the conscious resting state: A functional MRI study. Journal of Cognitive Neuroscience, 11, 80-93.

Binder, J.R., Frost, J.A., Hammeke, T.A., Bellgowan, P.S.F., Springer, J.A., Kaufman, J.N., \& Possing, E.T. (2000). Human temporal lobe activation by speech and nonspeech sounds. Cerebral Cortex, 10, 512-528.

Binder, J.R., Frost, J.A., Hammeke, T.A., Rao, S.M., \& Cox, R.W. (1996). Function of the left planum temporale in auditory and linguistic processing. Brain, 119, 1239-1247.

Binkofski, F., Amunts, K., Stephan, K.M., Posse, S., Schormann, T., Freund, H.-J., Zilles, K., \& Seitz, R.J. (2000). Broca’s region subserves imagery of motion: A combined cytoarchitectonic and fMRI study. Human Brain Mapping, 11, $273-285$.

Buchman, A.S., Garron, D.C., Trost-Cardonne, J.E., Wichter, M.D., \& Schwartz, D. (1986). Word deafness: One hundred years later. Journal of Neurology, Neurosurgery, and Psychiatry, 49, 499.

Burton, M.W., Small, S.L., \& Blumstein, S.E. (2000). The role of segmentation in phonological processing: An fMRI investigation. Journal of Cognitive Neurosci-ence, 12, 679-690.

Cabeza, R., \& Nyberg, L. (2000). Imaging cognition II: An empirical review of 275 PET and fMRI studies. Journal of Cognitive Neuroscience, 12, 1-47.

Caramazza, A., \& Shelton, J.R. (1998). Domain-specific knowledge systems in the brain: The animate-inanimate distinction. Journal of Cognitive Neurosci-ence, 10, 1-34.

Damasio, H., Grabowski, T.J., Tranel, D., Hichwa, R.D., \& Damasio, A.R. (1996). A neural basis of lexical retrieval. Nature, 380, 499-505.

Dehaene, S., Dupoux, E., Mehler, J., Cohen, L., Paulesu, E., Perani, D., van de Moortele, P.F., Lehéricy, S., \& Le Bihan, D. (1997). Anatomical variability in the cortical representation of first and second language. NeuroReport, 8, 3809-3815.

Démonet, J.F., Chollet, F., Ramsay, S., Cardebat, D., Nespoulous, J.L., Wise, R.J.S., Rascol, A., \& Frackowiak, R.S.J. (1992). The anatomy of phonological and semantic processing in normal subjects. Brain, 115, 1753-1768.

Démonet, J.F., Price, C., Wise, R.J.S., \& Frackowiak, R.S.J. (1994). Differential activation of right and left posterior sylvian regions by semantic and phonological tasks: A positron emission tomography study in normal human subjects. Neuroscience Letters, 182, 25-28.

De Renzi, E., \& Lucchelli, F. (1994). Are semantic systems separately represented in the brain? The case of living category impairment. Cortex, 30, 3-25.

E-Prime Beta 5.0 (2000). [Computer Software]. Pittsburgh, PA: Psychology Software Tools Inc.

Fiez, J.A. (1997). Phonology, semantics, and the role of inferior prefrontal cortex. Human Brain Mapping, 5, 79-83.

Foundas, A.L., Daniels, S.K., \& Vasterling, J.L. (1998). Anomia: Case studies with lesion localization. Neurocase, 4, $35-43$.

Frison, L., \& Pocock, S.J. (1992). Repeated measures in clinical trials: An analysis using mean summary statistics and it's implications for design. Statistics in Medicine, 11, 1685-1704.

Friston, K.J., Ashburner, J., Frith, C.D., Poline, J.B., Heather, J.D., \& Frackowiak, R.S.J. (1995). Spatial registration and normalisation of images. Human Brain Mapping, 3, 165-189.

Gathercole, S.E., Frankish, C.R., Pickering, S.J., \& Peaker, S. (1999). Phonotactic influences on shortterm memory. Journal of Experimental Psychology: Human Learning and Memory, 25, 84-95.

Gathercole, S.E., Hitch, G.J., Service, E., \& Martin, A.J. (1997). Phonological short-term memory and new word learning in children. Developmental Psychology, 33, 966-979.

Grasby, P.M., Frith, C.D., Friston, K.J., Bench, C., Frackowiak, R.S.J., \& Dolan, R.J. (1993). Functional mapping of brain areas implicated in auditory-verbal memory function. Brain, 116, 1-20.

Greenberg, J.H., \& Jenkins, J.J. (1964). Studies in the psychological correlates of the sound system of American English. Word, 20, 157-177.

Hart, J., \& Gordon, B. (1992). Neural subsystems for object knowledge. Nature, 359, 60-64.

Herholz, K., Pietrzyk, U., Karbe, H., Wurker, K., Wienhard, K., \& Heiss, W.D. (1994). Individual metabolic anatomy of repeating words demonstrated by MRI-guided positron emission tomography. Neuroscience Letters, 182, 47-50. 
Hickok, G. (2000). Speechperception,conduction aphasia, and the functional neuroanatomy of language. In Y. Grodzinsky, L. Shapiro, \& D. Swinney (Eds.), Language and the brain (pp. 87-104). San Diego, CA: Academic Press.

Hickok, G., Erhard, P., Kassubek, J., Helms-Tillery, A.K.,Naeve-Velguth, S., Strupp, J.P., Strick, P.L., \& Ugurbil, K. (2000). A functional magnetic resonance imaging study of the role of left posterior superior temporal gyrus in speech production: Implications for the explanation of conduction aphasia. Neuroscience Letters, 287, 156-160.

Hickok, G., \& Poeppel, D. (2000). Towards afunctional neuroanatomy of speech perception. Trends in Cognitive Sciences, 4 , $131-138$

Hillis, A.E., \& Caramazza, A. (1991). Category-specific naming and comprehension impairment: A double dissociation. Brain, $114,2081-2094$.

Hirano, S., Naito, Y., Okazawa, H., Kojima, H., Honjo, I., Ishizu, K., Yenokura, Y., Nagahama, Y., Fukuyama, H., \& Konishi, J. (1997). Cortical activation by monaural speech sound stimulation demonstrated by positron emission tomography. Experimental Brain Research, 113, 75-80.

Holmes, A., \& Friston, K. (1998). Generalisability, random effects and population inference. NeuroImage, 7(4), 754-754.

Howard, D., Patterson, K., Wise, R.J.S., Brown, W.D., Friston, K.J., Weiller, C., \& Frackowiak, R.S.J. (1992). The cortical localisation of the lexicons. Brain, 115, 1769-1782.

Hughes, H.C.,Darcey,T.M.,Barkan, H.I., Williamson, P.D., Roberts, D.W., \& Aslin, C.H. (2001). Responses of human auditory association cortex to the omission of an expected acoustic event. NeuroImage, 13, 1073-1089.

Hulme, C.,Maughan,S., \&Brown, G.D. (1991). Memory for familiar and unfamiliar words: Evidence for a long-term memory contribution to short-term memory span. Journal of Memory and Language, 30, 685- 701.

Jueptner, M., \& Weiller, C. (1995). Review: Does measurement of regional cerebral blood flow reflect syn-aptic activity? Implications for PET and fMRI. Neurolmage, 2, 148-56.

Jusczyk, P.W., Friederici, A.D., Wessels, J.M.I., Svenkerund, V.Y., \& Jusczyk, A.M. (1993). Infants' sensitivity to the sound patterns of native language words.Journalof MemoryandLanguage,32, 402-420.

Jusczyk, P.W., Luce, P.A., \& Charles-Luce, J. (1994). Infants' sensitivity to phonotactic patterns in the native language. Journal of Memory and Language, 33, 630-645.

Karbe, H., Herholz, K., Weber-Luxenburger, G., Ghaemi, M., \& Heiss, W.D. (1998). Cerebral networks and functional brain asymmetry: evidence from regional metabolic changes during word repetition. Brain and Language, 63, $108-121$.

Knott, R., Patterson, K., \& Hodges, J.R. (1997). Lexical and semantic binding effects in short-term memory: Evidence from semantic dementia. Cognitive Neuropsychology, 14, 1165-1216.

Luce, P.A., \& Pisoni, D.B. (1998). Recognising spoken words:Theneighbourhood activationmodel. Ear and Hearing, 19 , 1-36.

Mazoyer, B.M., Tzourio, N., Frak, V., Syrota, A., Murayama, N., Levrier, O., Salamon, G., Dehaene, S., Cohen, L., \& Mehler, J. (1993). The cortical representation of speech. Journal of Cognitive Neurosci-ence, 5, 467-479.

McCarthy,R.A., \&Warrington,E.K. (2001). Repeating without semantics: Surface dysphasia? Neurocase, 7, 77-87.

Muller, R.A., Kleinhans, N., \& Courchesne, E. (2001). Broca's area and the discrimination of frequency transitions: A functional MRI study. Brain and Language, 76, 70-76.

Musso, M., Weiller, C., Kiebel, S., Müller, S.P., Bülau, P., \& Rijntjes, M. (1999). Training-induced brain plasticity in aphasia. Brain, 122, 1781-1790.

Newman, R.S., Sawusch, J.R., \& Luce, P.A. (1996). Lexical neighbourhood effects in phonetic processing. Journal of Experimental Psychology: Human Perception and Performance, 23, 873-889.

Perani, D., Dehaene, S., Grassi, F., Cohen, L., Cappa, S.F., Dupoux, E., Fazio, F., \& Mehler, J. (1996). Brain processing of native and foreign languages. NeuroReport, 7, 2439-2444.

Petersen, S.E., Fox, P.T., Posner, M.I., Mintun, M., \& Raichle, M.E. (1988). Positron emission tomographic studies of the cortical anatomy of single-word processing. Nature, 331, 585-589.

Petersen, S.E., Fox, P.T., Posner, M.I., Mintun, M., \& Raichle, M.E. (1989). Positron emission tomo-graphic studies of the processing of single words. Journal of Cognitive Neuroscience, 1, 153-170.

Pitt, M.A., \& McQueen, J.M. (1998). Is compensation for coarticulation mediated by the lexicon? Journal of Memory and Language, 39, 347-370.

Price, C., Indefrey, P., \& Van Turennout, M. (1999). The neural architecture underlying the processing of written and spoken word forms. In C.M. Brown \& P. Hagoort (Eds.), The neurocognition of language (pp. 211-240). Oxford, UK: Oxford University Press. 
Published in: Cognitive neuropsychology (2002), vol. 19, iss. 4, pp. 343-360

Status: Postprint (Author's version)

Price, C.J., Wise, R.J.S., \& Frackowiak, R.S.J. (1994). Demonstrating the implicit processing of visually presented words and pseudowords. Journal of Cognitive Neuroscience, 6, 62-70.

Price, C.J., Wise, R.J.S., Warburton, E.A., Moore, C.J., Howard, D., Patterson, K., Frackowiak, R.S.J., \& Friston, K.J. (1996). Hearing and saying. The functional neuro-anatomy of auditory word processing. Brain, 119, 919-931.

Rapcsak, S., \& Rubens, A.B. (1990). Disruption of semantic influence on writing following a left prefrontal lesion. Brain and Language, 38, 334-344.

Sacchett, C., \& Humphreys, G.W. (1992). Calling a squirrel a squirrel but a canoe a wigwam: A category-specific deficit for artifactual objects and body parts. Cognitive Neuropsychology, 9, 73-86.

Scott, S.K., Blank, C., Rosen, S., \& Wise, R.J.S. (2000). Identification of a pathway for intelligible speech in the left temporal lobe. Brain, 123, 2400-2406.

Silveri, M.C., \& Gainotti, G. (1988). Interaction between vision and language in category-specific semantic impairment. Cognitive Neuropsychology, 5, 677-709.

Swick, D. (1998). Effects of prefrontal lesions on lexical processing and repetition priming: An ERP study. Cognitive Brain Research, 7, 143-157.

Talairach, J., \& Tournoux, P. (1988). Co-planar stereo-taxic atlas of the human brain. Stuttgart, Germany: Thieme Medical Publishers.

Tanaka, Y., Yamadori, A., \&Mori, E. (1987). Pureword deafness following bilateral lesions: a psycho-physiological analysis. Brain, 110, 381-403.

Tompkins, C.A., Baumgaertner, A., Lehman, M.T., \& Fassbinder, W. (2000). Mechanisms of discourse comprehension impairment after right hemisphere brain damage: Suppression in lexical ambiguity resolution. Journal of Speech, Language and Hearing Research, 43, 62-78.

Tompkins, C.A., Lehman, M.T., \& Fassbinder, W. (1998). Interpreting intended meanings after right hemisphere brain damage: An analysis of evidence, potential accounts, and clinical implications. Topics in Stroke Rehabilitation, 5, 29-47.

Tubach, J.L., \& Boë, L.J. (1990). Un corpus de transcription phonétique. France: Telecom.

Vitevitch, M.S. (1997). The neighborhood characteristics of malapropisms. Language and Speech, 40, 211- 228.

Vitevitch, M.S., \& Luce, P.A. (1998). When words compete: Levels of processing in perception of spoken words. Psychological Science, 9, 325-329.

Vitevitch, M.S., \& Luce, P.A. (1999). Probabilistic phonotactics and neighbourhood activationin spoken word recognition. Journal of Memory and Language, 40, 374-408.

Vitevitch, M.S., Luce, P.A., Charles-Luce, J., \& Kemmerer, D. (1997). Phonotactics and syllable stress: Implications for the processing of spoken nonsense words. Language and Speech, 40, 47-62.

Warrington, E.K., \&McCarthy,R.A. (1983).Category-specific access dysphasia. Brain, 106, 859-878.

Warrington, E.K., \& McCarthy, R.A. (1987). Categories of knowledge: Further fractionation and an attempted integration. Brain, $100,1273-1296$.

Warrington, E.K., \& Shallice, T. (1984). Category specific memory impairment. Brain, 107, 829-854.

Winkler, I., Karmos, G., \& Näätänen, R. (1996). Adaptive modeling of unattended acoustic environment reflected in the mismatch negativity event-related potential. Brain Research, 742, 239-252.

Wise, R.J.S., Chollet, F., Hadar, U., Friston, K.J., Hoffner, E., \& Frackowiak, R.S.J. (1991). Distribution of cortical neural networks involved in word comprehension and word retrieval. Brain, 114, 1803-1817.

Wise, R.J.S., Scott, S.K., Blank, S.C., Mummery, C.J., Murphy, K., \& Warburton, E.A. (2001). Separate neural subsystems within "Wernicke's area". Brain, 124, 83-95.

Zaidel, D.W.,Zaidel,E.,Oxbury,S.M.,\&Oxbury, J.M. (1995). The interpretation of sentence ambiguity in patients with unilateral focal brain surgery. Brain and Language, 51, 458-468.

Zatorre, R.J., Evans, A.C., Meyer, E., \& Gjedde, A. (1992). Lateralisation of phonetic and pitch discrimination in speech processing. Science, 256, 846-849.

Zatorre, R.J., Meyer, E., Gjedde, A., \& Evans, A.C. (1996). PET studies of phonetic processing ofspeech: Review, replication and reanalysis. Cerebral Cortex, 6, 21-30. 
Published in: Cognitive neuropsychology (2002), vol. 19, iss. 4, pp. 343-360 Status: Postprint (Author's version) 